\title{
Association of cardiac troponin I with prothrombotic alterations in atrial fibrillation
}

\author{
Paweł T. Matusiki, ${ }^{1,2}$, Shannon M. Prior ${ }^{3}$, Saulius Butenas ${ }^{3}$, Barbara Małecka ${ }^{1,2}$, \\ Jacek Lelakowski ${ }^{1,2}$, Anetta Undas ${ }^{2,4}$ \\ 'Department of Electrocardiology, John Paul II Hospital, Krakow, Poland \\ ${ }^{2}$ Institute of Cardiology, Jagiellonian University Medical College, Krakow, Poland \\ ${ }^{3}$ Department of Biochemistry, University of Vermont, Colchester, VT, United States \\ ${ }^{4}$ Krakow Centre for Medical Research and Technology, John Paul II Hospital, Krakow, Poland
}

\section{INTRODUCTION}

Atrial fibrillation (AF) increases the risk of stroke and systemic thromboembolism. A hypercoagulable state in AF is reflected by elevated von Willebrand factor (VWF), D-dimer, and thrombin generation (TG), as well as increased platelet activation [1]. The usefulness of several biomarkers in stroke and bleeding risk prediction among AF patients, in particular $\mathrm{N}$-terminal pro-B-type natriuretic peptide (NT-proBNP) and high-sensitivity cardiac troponin I (cTnl-hs), has been demonstrated, although practical implications of this strategy are uncertain [2].

We sought to assess the relations of four such markers: cTnl-hs, cystatin C, vWF, and NT-proBNP, with prothrombotic alterations in AF patients.

\section{METHODS}

We investigated 65 patients with AF free of myocardial infarction or venous thromboembolism within the previous three months, with creatinine clearance $\geq 15 \mathrm{~mL} / \mathrm{min}$, and without liver cirrhosis, known cancer, or acute infection. Detailed methodology of the study was presented previously [3]. Vitamin $\mathrm{K}$ antagonists and low-molecular-weight heparin (the last dose $\geq 12 \mathrm{~h}$ ) were discontinued before the blood draw. The study was approved by the Bioethical Committee, and patients gave informed consent.

Venous blood samples were taken with minimal stasis, and routine blood tests were performed by standard laboratory techniques. Electrochemiluminescence immunoassay (Roche Diagnostics, Mannheim, Germany) was used to measure NT-proBNP levels. The ARCHITECT i1000SR and ARCHITECT ci8200 (Abbott Laboratories, Abbott Park, IL, USA) were used to assess cTnl-hs and cystatin C levels, respectively. Latex immunoassay using a STAR coagulation instrument (Diagnostica Stago, Asnières, France) was used to measure vWF antigen.

Assessment of TG profile was performed as described [4]. Briefly, corn trypsin inhibitor was added to citrated plasma (a final concentration of $0.1 \mathrm{mg} / \mathrm{mL}$ ), and then samples (80 $\mu \mathrm{L}$ each) were mixed with relipidated tissue factor to achieve a final concentration of $5 \mathrm{pM}$ and with a $2.5 \mathrm{mM}$ Z-GGR-AMC/90 mM CaCl 2 solution in Hepes-buffered saline to achieve a $417 \mu \mathrm{M}$ and $15 \mathrm{mM}$ final concentration, respectively, followed by incubation at $37^{\circ} \mathrm{C}$ for $3 \mathrm{~min}$. Lastly, a phospholipid vesicles solution in Hepes-buffered saline was added to samples to achieve $20 \mu \mathrm{M}$ final concentration, thereby initiating TG. Hydrolysis of the 7-amino-4-methylcoumarin substrate at $370 \mathrm{~nm}$ excitation and $460 \mathrm{~nm}$ emission wavelengths was followed for $3600 \mathrm{~s}$. A calibration curve built by sequential dilutions of human thrombin into pooled multi-donor plasma from healthy individuals was used to convert substrate hydrolysis to thrombin concentration. We used the BioTek Synergy 4 plate reader and Gen5 plate reader software for analysis (BioTek, Winooski, VT, USA).

\section{Statistical analysis}

Quantitative variables were presented as the mean (standard deviation) or median (interquartile range). Normal distribution was assessed using the Shapiro-Wilk test. Quantitative variables were compared using Student t-test or Mann-Whitney $U$ test, and correlations were tested using a linear Pearson correlation or Spearman's rank test, as appropriate. We divided patients into three groups based on tertiles of cTnl-hs levels, and into four groups based on the $\mathrm{CHA}_{2} \mathrm{DS}_{2}-\mathrm{VASC}$ score. Normally distributed data in three or more groups 

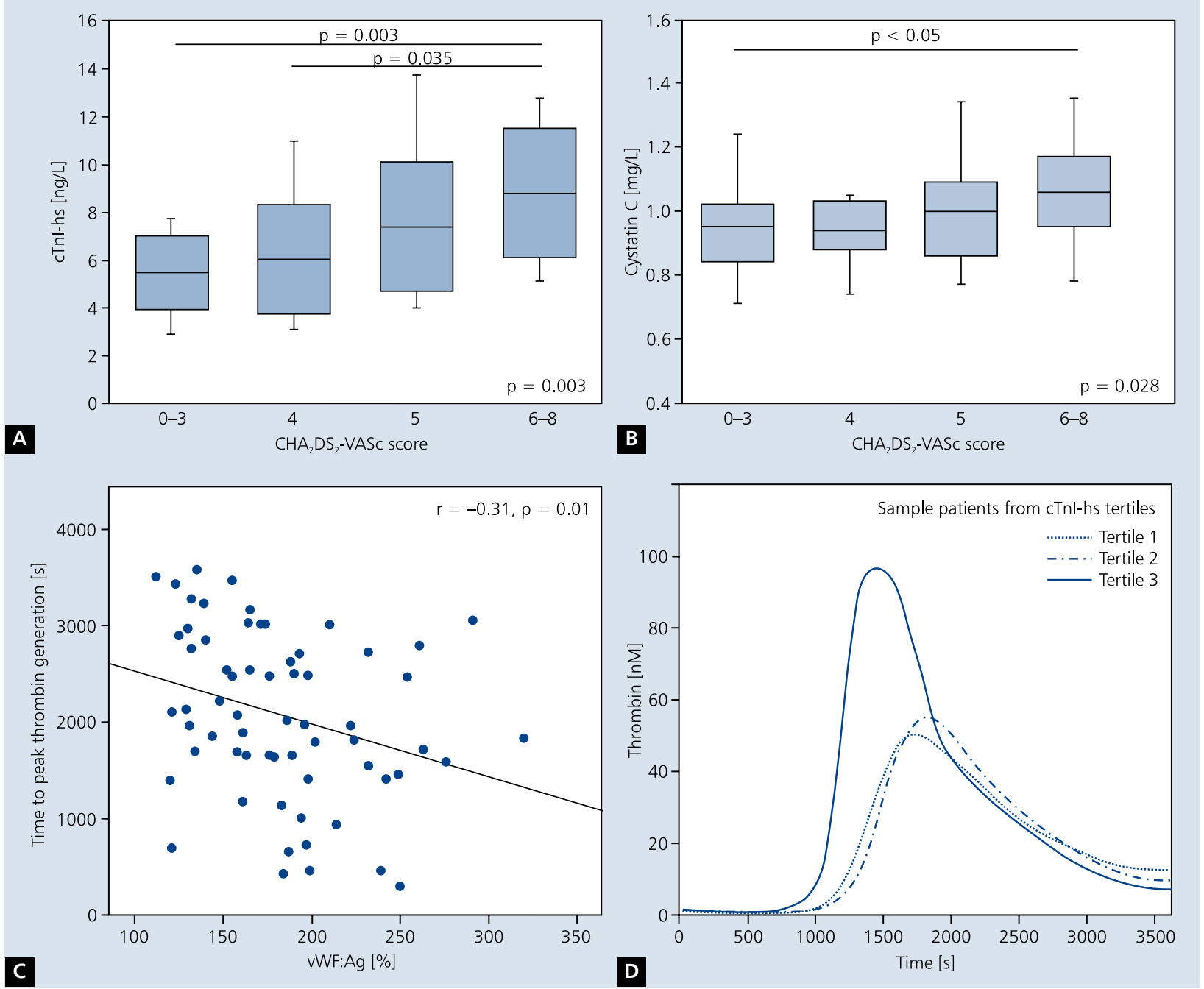

Figure 1. Associations of $\mathrm{CHA}_{2} \mathrm{DS}_{2}$-VASc score with high-sensitivity cardiac troponin I (cTnl-hs; $\mathbf{A}$ ) and cystatin C (B) in patients with atrial fibrillation (AF). Correlation between time to peak thrombin generation and von Willebrand factor (VWF) in patients with $\mathrm{AF}(\mathbf{C}) ; \mathrm{Ag}$ - antigen. Thrombin generation among sample patients from different cTnl-hs tertiles (D). Values are presented as mean (standard deviation), maximum and minimum (for cTnl-hs), or median (interquartile range), maximum and minimum (for cystatin C)

were compared using one-way ANOVA (in homogeneity of variance) or Welch ANOVA (otherwise). Tukey's HSD test was used to assess pairwise comparisons. For comparison of non-normally distributed variables in three or more groups, the Kruskal-Wallis test was used, and for pairwise comparisons the Kruskal-Wallis test with the Bonferroni correction was used. Qualitative variables were described by number (frequency) and compared by $\chi^{2}$ test or Fisher exact test. To assess predictors of peak thrombin concentration we performed linear regression analysis. $\mathrm{R}^{2}$ was calculated, and assessment of model adequacy using $\mathrm{F}$ test was performed. $P$ values of $<0.05$ were considered statistically significant. IBM SPSS Statistics (version 24.0, IBM Corp., Armonk, NY, USA) was used to perform statistical analysis.

\section{RESULTS AND DISCUSSION}

The current study group included 65 patients aged $69.8 \pm 9.9$ years (41.5\% women), who had mostly permanent AF (53.8\%) (Suppl. Table 1 - see journal website). $\mathrm{CHA}_{2} \mathrm{DS}_{2}$-VASc score $\geq 2$ was observed in $93.8 \%$ of patients.

Patients in the third cTnl-hs tertile were older than those in the first and second tertiles (all $p<0.005$ ). Patients in the first cTnl-hs tertile had a higher haemoglobin concentration than those in the third cTnl-hs tertile $(p=0.02)$ (Suppl. Table 2 - see journal website). In the whole group cystatin $C$ was correlated with cTnl-hs $(r=0.34, p=0.005)$, but not with NT-proBNP $(r=0.12, p=0.33)$.

$\mathrm{CHA}_{2} \mathrm{DS}_{2}$-VASc score was positively correlated with cTnl-hs $(R=0.47, p<0.001)$ and cystatin C $(r=0.39$, 
$p=0.001)$, with a similar trend for NT-proBNP $(R=0.22$, $p=0.07$ ). cTnl-hs and cystatin C (Fig. 1A, B) were higher in subjects with the highest $\mathrm{CHA}_{2} \mathrm{DS}_{2}$-VASc score when compared to patients with the lowest $\mathrm{CHA}_{2} \mathrm{DS}_{2}$-VASc score. No such associations were observed for NT-proBNP, vWF, creatinine, or TG parameters. There was no correlation between vWF and $\mathrm{CHA}_{2} \mathrm{DS}_{2}$-VASc score, cTnl-hs, cystatin C, or NT-proBNP (data not shown).

Analysis of TG showed that lag phase and endogenous thrombin potential did not correlate with cTnl-hs, NT-proBNP, or cystatin $\mathrm{C}$. Time to peak thrombin generation was correlated with vWF (Fig. 1C) and tended to correlate with cTnl-hs $(r=-0.22, p=0.08)$, but not with NT-proBNP or cystatin C. Importantly, peak thrombin concentration correlated with cTnl-hs and was associated with cTnl-hs tertiles $(\mathrm{R}=0.37, \mathrm{p}=0.003$, Fig. 1D) but not with NT-proBNP, cystatin $\mathrm{C}$, or vWF. Peak thrombin concentration was not related to glomerular filtration rate (GFR) and age $(r=0.04$, $p=0.73$ and $r=0.10, p=0.44$, respectively). In linear regression analysis peak thrombin concentration was predicted by cTnl-hs (unstandardised coefficient B: 17.09; 95\% confidence interval $[\mathrm{Cl}]: 0.06-34.11, \mathrm{p}=0.049)$ but not GFR (B: $0.43 ; 95 \% \mathrm{Cl}:-2.02-2.88 ; \mathrm{p}=0.73$ ) or age (B: $1.84 ; 95 \% \mathrm{Cl}:-2.86-6.54 ; \mathrm{p}=0.44)$. Peak thrombin concentration was predicted by cTnl-hs (B: $27.30 ; 95 \% \mathrm{Cl}$ : 7.15-47.45; $\mathrm{p}=0.009)$, after inclusion of GFR in the model (B: $2.55 ; 95 \% \mathrm{Cl}:-0.26-5.37 ; \mathrm{p}=0.074), \mathrm{R}^{2}=0.11, \mathrm{~F}=3.73$, $\mathrm{p}=0.029$.

Our study shows that peak thrombin concentration is associated with cTnl-hs in AF patients, suggesting that the most sensitive TG parameter is associated with processes leading to myocardial injury, strain, or ischaemia in this common arrhythmia. This sensitivity of peak thrombin, but not endogenous thrombin potential, is best reflected by the observation that in patients with acute coronary syndromes at admission and six months later the sole elevated TG marker is peak thrombin when compared to controls [5]. Peak thrombin generated, together with shorter times to maximum levels, are well-established features of hypercoagulable states and may be useful in the assessment of bleeding and thromboembolic risk [6]. Our preliminary study suggests that cardiac troponins better reflect TG in AF compared with other biomarkers. In AF elevation of cTnl-hs is related to cardiac disease, including heart failure and coronary artery disease, the risk factors for stroke in $\mathrm{AF}$, being associated with the severity of myocardial ischaemia and/or injury [7, 8]. Troponin release may also occur during tachyarrhythmias and bradyarrhythmias frequently observed in patients with $\mathrm{AF}$ [9].

We confirmed that cTnl-hs and renal function parameters are associated with $\mathrm{CHA}_{2} \mathrm{DS}_{2}-\mathrm{VASc}$ score $[10,11]$ and that higher AF burden is associated with elevated troponin levels [7].

Our study supports biomarker-based risk stratification among patients with $\operatorname{AF}[2,12]$. Current results and our previous observations indicate that cardiac troponin and NT-proBNP may be implicated in similar pathogenic pathways leading to faster and/or enhanced TG, which significantly contributes to a hypercoagulable state in AF [3]. These two biomarkers and associated pathways may be common with those noted in ischaemic stroke [13].

The current study has several limitations. First, the study group was small. Secondly, TG can be determined using various assays, including the use of different concentrations of reagents in calibrated automated thrombograms. We did not measure anti-Xa activity in our study. It is unclear whether anticoagulated AF patients show similar associations between TG variables and other biomarkers.

The current study provides insights into relations of circulating biomarkers with thromboembolic risk and thrombin generation in patients with AF.

\section{Acknowledgements}

This work was supported by the National Institutes of Health (TACTIC grant UM1 HL120877 [to S.B.]).

\section{Conflict of interest: none declared}

\section{References}

1. Drabik L, Wołkow P, Undas A. Denser plasma clot formation and impaired fibrinolysis in paroxysmal and persistent atrial fibrillation while on sinus rhythm: association with thrombin generation, endothelial injury and platelet activation. Thromb Res. 2015; 136(2): 408-414, doi: 10.1016/j.thromres.2015.05.028, indexed in Pubmed: 26048399.

2. Hijazi Z, Lindbäck J, Alexander JH, et al. The ABC (age, biomarkers, clinical history) stroke risk score: a biomarker-based risk score for predicting stroke in atrial fibrillation. Eur Heart J. 2016; 37(20): 1582-1590, doi: 10.1093/eurheartj/ehw054, indexed in Pubmed: 26920728.

3. Matusik PT, Matusik PS, Kornacewicz-Jach Z, et al. Elevated NT-proBNP is associated with unfavorably altered plasma fibrin clot properties in atrial fibrillation. Int J Cardiol. 2017; 243: 244-250, doi: 10.1016/j.ijcard.2017.05.060, indexed in Pubmed: 28571619.

4. Goldman S, Prior SM, Bembenek JP, et al. Activation of blood coagulation and thrombin generation in acute ischemic stroke treated with rtPA. J Thromb Thrombolysis. 2017; 44(3): 362-370, doi: 10.1007/s11239-017-1544-7, indexed in Pubmed: 28879581.

5. Skeppholm M, Kallner A, Malmqvist K, et al. Is fibrin formation and thrombin generation increased during and after an acute coronary syndrome? Thromb Res. 2011; 128(5): 483-489, doi: 10.1016/j.thromres.2011.03.011, indexed in Pubmed: 21496882.

6. Hemker HC, Al Dieri R, De Smedt E, et al. Thrombin generation, a function test of the haemostatic-thrombotic system. Thromb Haemost. 2006; 96(5): 553-561, indexed in Pubmed: 17080210.

7. Hijazi Z, Siegbahn A, Andersson U, et al. Comparison of cardiac troponins I and T measured with high-sensitivity methods for evaluation of prognosis in atrial fibrillation: an ARISTOTLE substudy. Clin Chem. 2015; 61(2): 368-378, doi: 10.1373/clinchem.2014.226936, indexed in Pubmed: 25451868

8. Osmancik P, Louckova A. Biomarkers of apoptosis, inflammation, and cardiac extracellular matrix remodelling in the prognosis of heart failure. Kardiol Pol. 2017; 75(4): 295-305, doi: 10.5603/KP.a2016.0154, indexed in Pubmed: 27747854 
9. Matusik PT, Tomala I, Piekarz I, et al. Thyroid goiter: implications for implantation of cardiovascular implantable electronic devices and cannulation of the superior vena cava confluence. Pol Arch Med Wewn. 2016; 126(6): 432-434, doi: 10.20452/pamw.3459, indexed in Pubmed: 27362396.

10. Hijazi Z, Siegbahn A, Andersson U, et al. High-Sensitivity troponin I for risk assessment in patients with atrial fibrillation: insights from the apixaban for reduction in stroke and other thromboembolic events in atrial fibrillation (ARISTOTLE) trial. Circulation. 2013; 129(6): 625-634, doi: 10.1161/circulationaha.113.006286.

11. Hohnloser SH, Hijazi Z, Thomas L, et al. Efficacy of apixaban when compared with warfarin in relation to renal function in patients with atrial fibrillation: insights from the ARISTOTLE trial. Eur Heart J. 2012; 33(22): 2821-2830, doi: 10.1093/eurheartj/ehs274, indexed in Pubmed: 22933567.

12. Kızılırmak F, Gokdeniz T, Gunes HM, et al. Myocardial injury biomarkers after radiofrequency catheter and cryoballoon ablation for atrial fibrillation and their impact on recurrence. Kardiol Pol. 2017; 75(2): 126-134, doi: 10.5603/KP.a2016.0089, indexed in Pubmed: 27221959.

13. Etgen T, Baum H, Sander K, et al. Cardiac troponins and N-terminal pro-brain natriuretic peptide in acute ischemic stroke do not relate to clinical prognosis. Stroke. 2005; 36(2): 270-275, doi: 10.1161/01.STR.0000151364.19066.a1, indexed in Pubmed: 15604421.

Cite this article as: Matusik PT, Prior SM, Butenas S, et al. Association of cardiac troponin I with prothrombotic alterations in atrial fibrillation. Kardiol Pol. 2018; 76(7): 1106-1109, doi: 10.5603/KP.2018.0134. 